\title{
Optical coatings - precision engineering on non-flat surfaces
}

\author{
Tammo Böntgen, Marc Neufert, Lars Jensen \\ Laser Zentrum Hannover e.V. - Hollerithallee 8, 30419 Hannover, Germany
}

\begin{abstract}
Manufacturing of optical coatings is about the precise deposition of material and its control on the nanometer scale. This has to be achieved for a stack of multiple layers with a total thickness that can reach several micrometer. In addition, this has to be maintained for the complete functional area. In this contribution we demonstrate a process capable of delivering these accuracies not only to flat but also to curved surfaces.
\end{abstract}

\section{Introduction}

Functional coatings are a fundamental part of most optical instruments. They enhance performance by supressing unwanted reflections, provide high reflective mirrors or deliver complex functionalities like radiation filtering or polarization dependent beam splitting. The fabrication of these coatings requires the deposition of alternating layer materials. While anti-reflective coatings are often comprised of only two or four layers the number of layers quickly rises as the desired functionality becomes more complex. When considering a bandpass or edge filter the number of layers increases and the total thickness of these coatings can exceed tenth of micrometers. This puts a high demand on the precision of the manufacturing process as the final spectral performance of a coating is brought about by the combined contributions from every layer. These are in turn defined by the exact thickness of the individual layers. With a process in place that can deliver the needed accuracy it is now also necessary to deliver it to the desired surface requiring also a lateral uniformity. On flat surfaces state of the art coating processes can deliver the needed performance and lateral uniformity. The coating of lenses and other curved substrates presents an additional challenge. Due to the curvature a coating applied without additional means will be inhomogeneous. This will in turn lead to a varying optical performance over the lens surface. To overcome this, two things need to be added. First an additional rotation of the curved substrate on the (rotating) calotte is necessary. This will ensure a rotational symmetry of the coating and eliminate any shadowing effects that the surface might cause. The second is a shaper mask that is specifically tailored to the lens surface that is coated. Production and verification of the mask can be time consuming and difficult. We will show that a system of specialized jigs can be used to overcome these difficulties and allow an easy verification of the mask and process. Moreover, this can be done using simple witness samples without the need for an actual lens to test the process. The jig also allows for having several witness samples in a coating run that unravel the optical performance of the coating at different positions on the lens. The ease of analysis of these witness samples adds control and reliability to the process.

\section{Coating system setup}

All coatings were fabricated using custom build ion beam sputtering (IBS) plants. The IBS coating technology is known to have a high stability and repeatability. With that it is well suited for the deposition of complex coatings. Non the less a highly accurate process control system is needed to harness these capabilities. A key component here is a broadband optical monitor (BBM). The BBM will continuously measure the transmission of the optic being coated in a wide spectral range during the deposition process. The measured spectrum is then compared to the expected theoretical curve. This allows the calculation of the actual layer thickness and growth rate at any time during the process. This delivers the needed precision and is used to control and terminate the deposition process. The achieved accuracies for an individual layer are in the range of $\sim 1 \mathrm{~nm}$. These accuracies are routinely established for flat surfaces. More complex geometries however require additional steps. First the rotational symmetry of a substrate needs to be exploited. This is done by using a planetary rotation system. In this each substrate is placed in a separate fixture that rotates around its own axis. All fixtures than rotate around a common central axis. The complex movement in the coating chamber is necessary because in sputtering systems the (sputtered) coating material is not distributed evenly in the chamber. A single rotation ensures an azimuthal uniforms distribution for flat surfaces. For curved surfaces another rotation is necessary to compensate for the substrate geometry. While the coating is now distributed evenly along an azimuthal trajectory it is still non-uniform along the radius. In a last step, a shadow mask is now added to the system. This mask blocks a portion of the coating material from reaching defined radii on the substrate surface. While the setup of the plant does in general not depend on the exact geometry of the substrate surface the shadow mask has to be tailored carefully to its geometry. Keeping in mind the

\footnotetext{
* Corresponding author: t.boentgen@1zh.de
} 
necessary accuracy for complex coatings the development of the masks is essential. To allow this, a systematic approach was developed to iteratively find masks for lens shaped surfaces. For each individual surface a dedicated jig is manufactured that can fit several flat witness samples. The samples are placed in the jig such that each represents a defined spot on the surface of the substrate that is to be coated. This is true for the position the surface and the inclination angle of the surface (Fig. 1). When measuring these samples a map of the uniformity of a coating on the surface of the lens can be created. The data collected from these measurements is then used to create a shadow mask that ensures uniformity for the specific surface. Initially the uniformity of a surface with no mask is used as input to calculate an initial mask. In an iterative process this mask is then optimized to fulfill the performance needs.

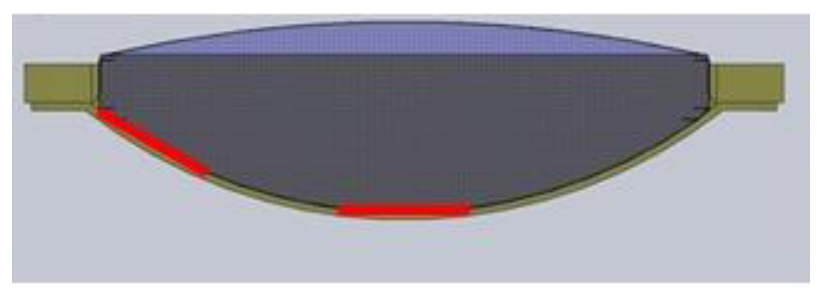

Fig. 1. Schematic view of witness sample positions. A cut of a lens is depicted in blue and two possible positions for witness samples in red. Any position along the lens surface can be chosen.

\section{Experimental}

Using the described procedure a total of 32 different surfaces (16 lenses) were processed and masks for uniformity needs of at least $1 \%$ were fabricated. The coated lenses have diameters from $\varnothing 50 \mathrm{~mm}$ to $\varnothing 80 \mathrm{~mm}$ and feature radii of curvature from $45 \mathrm{~mm}$ to $3300 \mathrm{~mm}$. The vast number of surfaces demonstrates the versatility of our approach. This allows the deposition of coatings which are not sensitive to the distribution of the angle of incidence (e.g. angular tolerant anti-reflective coatings) across the surface.

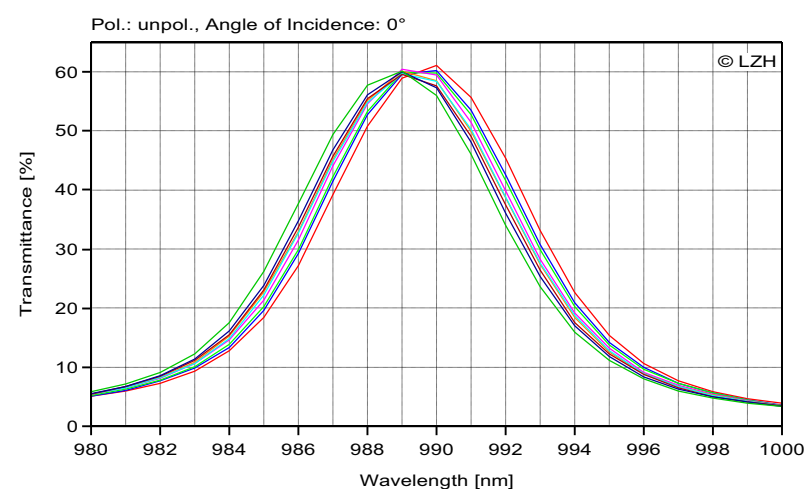

Fig. 2. Filter centered at $990 \mathrm{~nm}$. All curves are from measurements taken on positions from center to the edge representing a distance of $40 \mathrm{~mm}$.

For more complex coatings a better uniformity is needed. To further asses the capabilities of this approach a designated surface was chosen for which the uniformity was increased beyond the $1 \%$ mark. Ultimately on an area of $\varnothing 80 \mathrm{~mm}$ a uniformity of $0,1 \%$ was achieved (Fig.2). For coatings with steep edges in the spectral performance (e.g. Bandpass filters) another effect has to be considered. When the coating is to be applied on a non flat substrate the angle of incidence (AOI) is usually not constant over the substrate. Depending on the curvature it can vary substantially. A coating will, to a first degree, be shifted spectrally if viewed at different angles of incidence. A larger angle of incidence will shift the coating to shorter wavelength. Using a dedicated shadow mask this issue can be addressed because the spectral performance of the coating will also shift if the coating thickness is spatially inhomogeneous. It is assumed, that the uniformity will affect each layer in the coating in the same way. Thus areas that deviate from the target $(+-0 \%)$ will be red shifted (thicker / positive uniformity) or blue shifted (thinner / negative uniformity) with respect to the target. Since the AOI and its spread are defined by the application they can usually not be altered. However, the uniformity of the coating can be used to compensate the shift caused by the AOI spared. This is done by computing the non-uniformity necessary to compensate the shift caused by the AOI and subsequent fabrication of a shadow mask. This process is essentially the same as the one used to find masks that result in homogenous coatings. The coating deposited with the use of the fabricated shadow mask is shown in Figure3. Measurements were done on the witness samples. Each sample is viewed at its specific AOI that the corresponding part of the lens will be subjected to. The spectral shift of the coating is significantly reduced resulting in a fixed edge for any position and AOI on the lens.

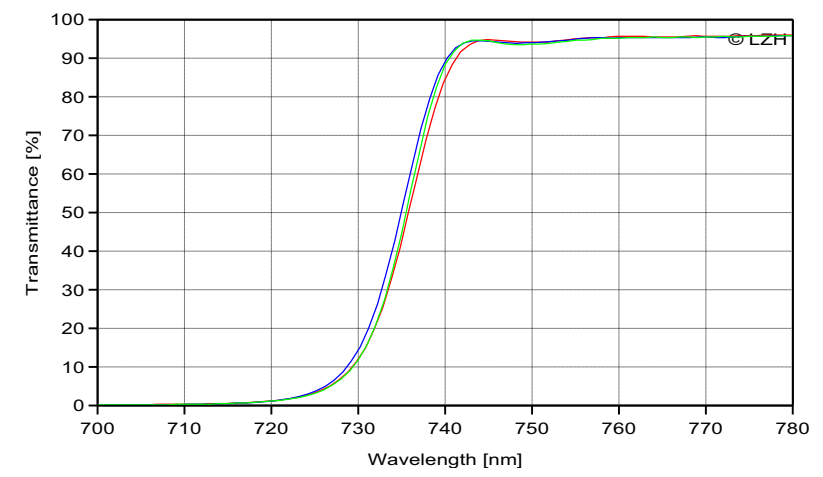

Fig. 3. Measured performance of the coating on the lens. Measurement was done on witness samples. Each sample is viewed at its specific AOI.

\section{Summary}

We have demonstrated a versatile approach for the coating of non-flat substrates that can increase the performance of simpler AR coatings but more importantly opens the way for the application of complex coatings with high demands on the coating process ultimately increasing freedom and allowing more compact optical designs. 